\title{
Vizille, Musée de la Révolution française
}

Nouvelles acquisitions

Alain Chevalier

\section{(2) OpenEdition}

Journals

Édition électronique

URL : https://journals.openedition.org/ahrf/8113

DOI : 10.4000/ahrf.8113

ISSN : 1952-403X

Éditeur :

Armand Colin, Société des études robespierristes

Édition imprimée

Date de publication : 1 décembre 2006

Pagination : 121-132

ISSN : 0003-4436

Référence électronique

Alain Chevalier, "Vizille, Musée de la Révolution française », Annales historiques de la Révolution française [En ligne], 346 | Octobre/Décembre 2006, mis en ligne le 01 décembre 2009, consulté le 24 avril 2022. URL : http://journals.openedition.org/ahrf/8113; DOI : https://doi.org/10.4000/ahrf.8113

Ce document a été généré automatiquement le 24 avril 2022

Tous droits réservés 


\section{Vizille, Musée de la Révolution française}

Nouvelles acquisitions

\section{Alain Chevalier}

Jean-Jacques Taillasson (Bordeaux, 1745 - Paris, 1809).

La Liberté ramenant aux peuples la Justice et la Vertu.

Vers 1794-1795.

Huile sur toile.

+H. 0,34 m. ; L. 0,50 m

Inv. MRF 2003-11.

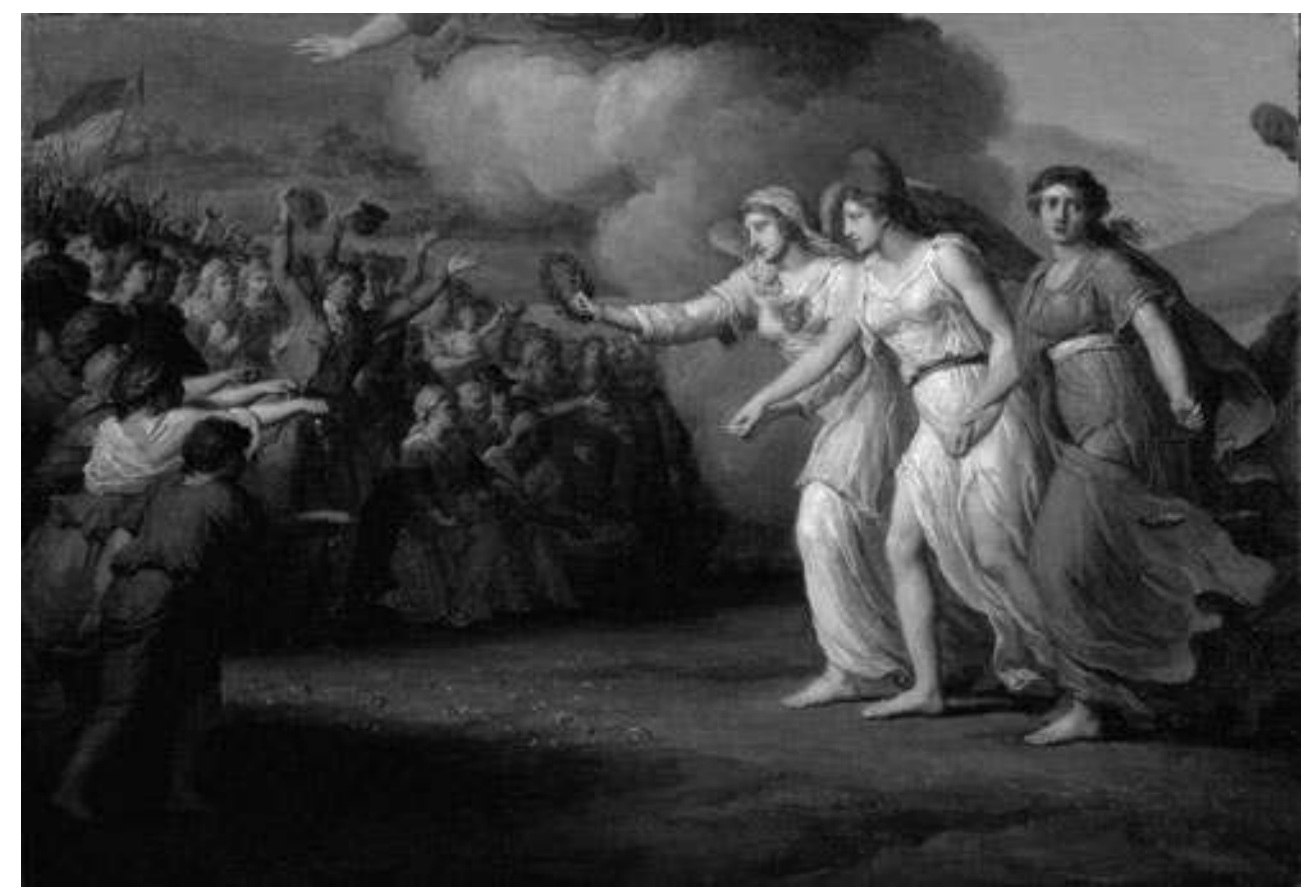

1 Nombre d'œuvres présentées au concours de l'an II (1794) ne sont pas localisées. Au hasard du marché, certaines d'entre elles, dont l'existence était réduite à une simple 
mention, réapparaissent parfois, comme l'esquisse proposée par le célèbre peintre bordelais Jean-Jacques Taillasson sous le titre : La Liberté ramenant aux peuples la Justice et la Vertu. Les figures allégoriques sont très proches de la Sapho du tableau du Musée des beaux-arts de Brest (Sapho ne pouvant se faire aimer du jeune Phaon se précipite du rocher de Leucate dans la mer) et de la Rodogune du tableau du Museum of Fine Arts de Houston (Mort de Cléopâtre tirée de "Rodogune»), tous deux exposés au Salon de 1791. Outre la qualité picturale raffinée de l'esquisse, un aspect iconographique est essentiel dans cette œuvre : la représentation très rare du peuple dans sa diversité sociale et raciale (présence d'un couple de noirs). Située entre Pauline, femme de Sénèque, ne voulant pas survivre à son mari, tableau d'avril 1793 (Musée du Louvre) et Léandre et Héro, son chefd'œuvre du Salon de 1798 (Musée des beaux-arts de Bordeaux), cette esquisse est un jalon important et inédit dans la période révolutionnaire de Taillasson. La restauration a révélé une coupure de la toile en haut et à droite laissant deviner respectivement une Renommée et un Hercule brandissant sa massue. En se référant au titre qui est celui de l'époque, cette intervention est sans doute due à l'artiste, désireux de resserrer sa composition.

Nicolas-Antoine Taunay (Paris, 1755 - Paris, 1830). Installation d'une statue de la République.

Vers 1793.

Huile sur toile.

H. 0,28 m. ; L. 0,395 m.

Inv. MRF 2004-5.

Achat avec le concours du FRAM Rhône-Alpes.

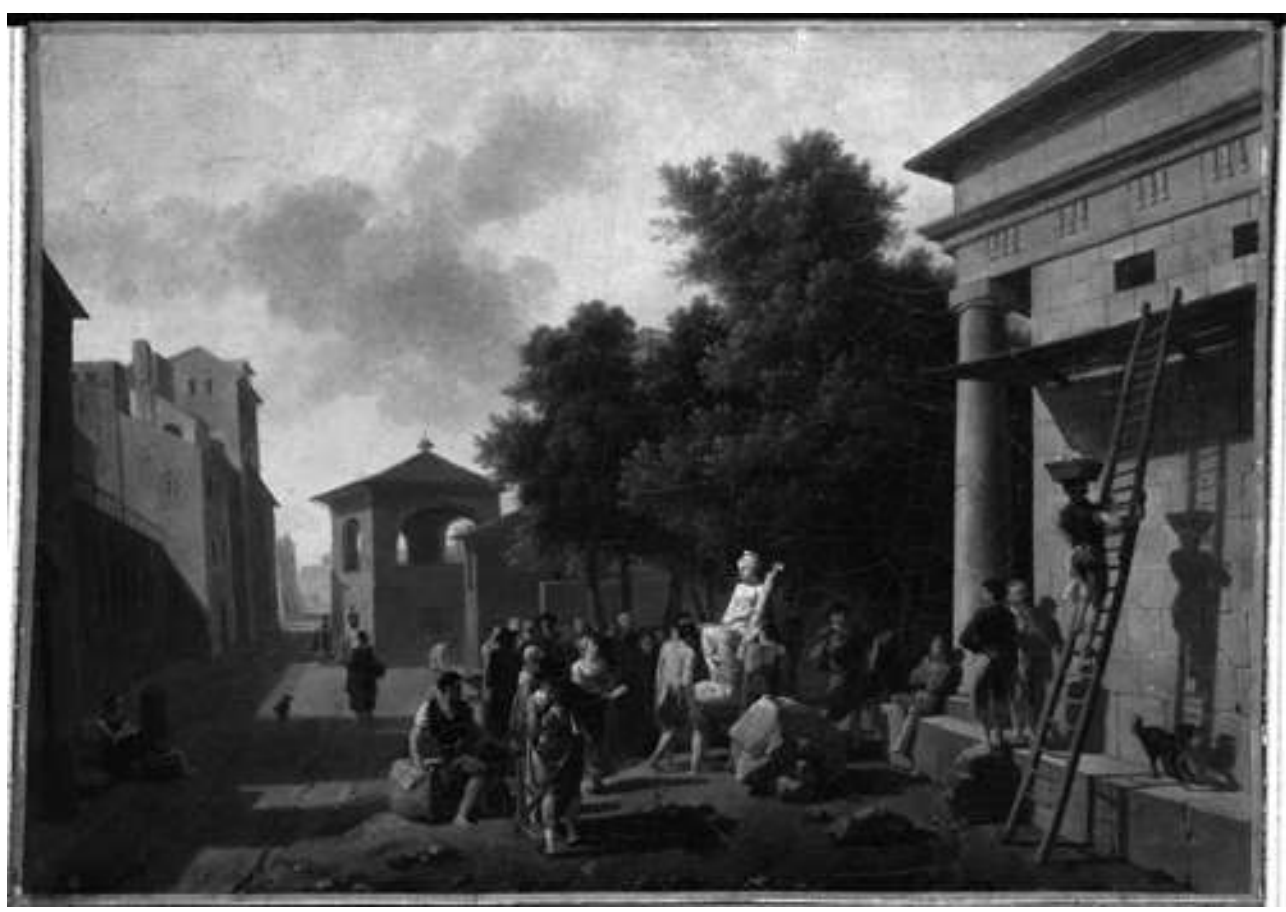

2 L'installation d'une statue de la République dans un édifice situé au cœur d'une petite ville imaginaire d'Italie (peut-être en Toscane ou en Campanie) est un sujet quasiment unique pendant la période révolutionnaire et surtout l'un des très rares tableaux de Taunay évoquant l'actualité politique. Après un séjour à l'Académie de France à Rome de 1784 à 1787, Nicolas-Antoine Taunay, peintre de genre et de paysage aux ambitions de peintre d'histoire, occupa rapidement dès son arrivée une place non négligeable sur 
la scène artistique parisienne. La Révolution ne freina guère son activité puisqu'il fréquenta très régulièrement et avec un succès grandissant le Salon à partir de 1789 . Son engagement en faveur de la Révolution est néanmoins faible malgré sa participation au prix d'encouragement de 1791 et au concours de l'an II, à l'occasion desquels il obtint des prix. De 1793 à 1795, il préféra quitter Paris pour Montmorency. Le métier raffiné de Taunay, son éclectisme et sa capacité de réaction à la modernité picturale de l'époque lui permettaient d'être en phase avec les attentes de la clientèle dont le goût et les origines avaient changé.

Ernst Meisel (Lichte,Thuringe, 1838 - Munich, 1895). La Séparation de Louis XVI de sa famille au Temple.

1873.

Huile sur toile.

H. 1,11 m. ; L. 1,82 m.

Inv. MRF 2004-13.

Achat avec le concours du FRAM Rhône-Alpes.

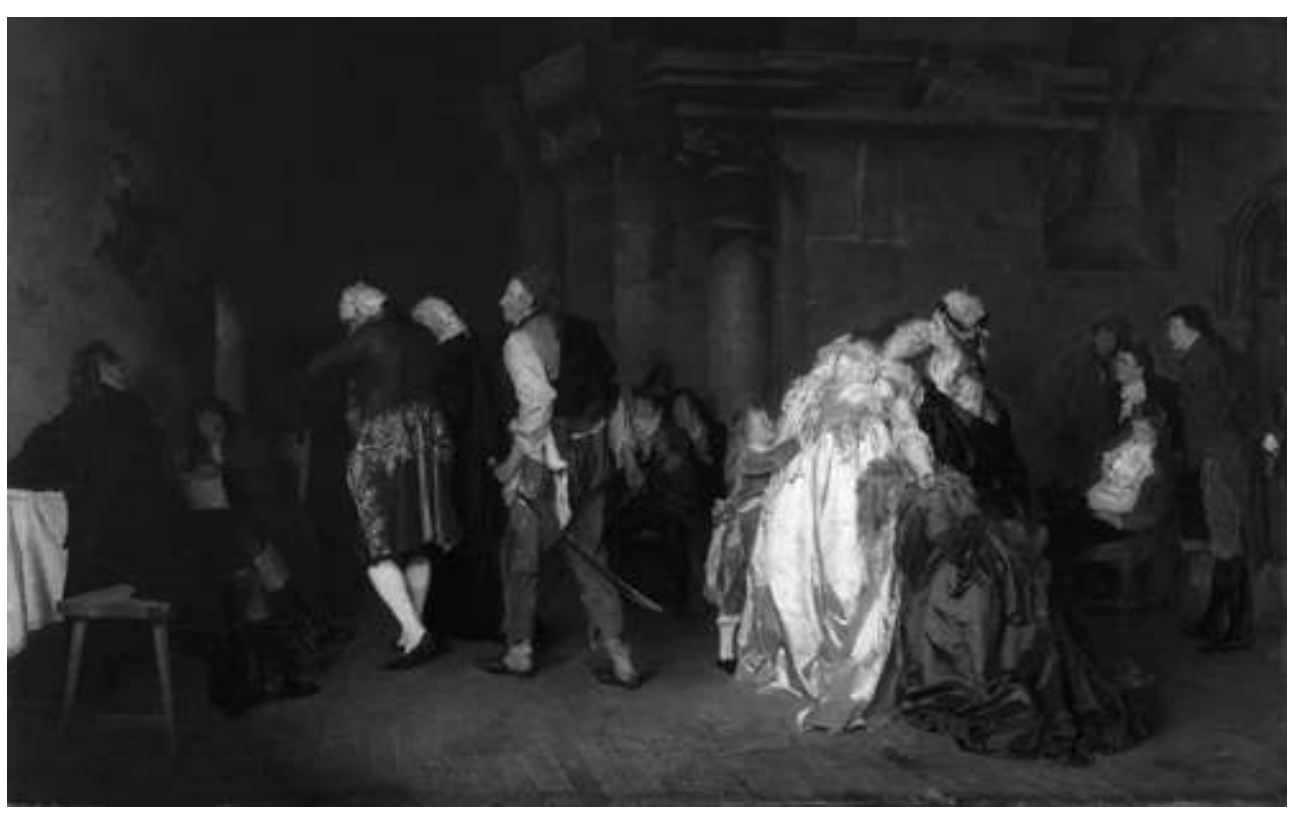

Une des préoccupations constantes du musée est d'ouvrir largement son champs d'investigation à l'Europe et aux États-Unis, en particulier en ce qui concerne sa politique d'acquisition. Si la vision rétrospective de la Révolution française au XIX ${ }^{\mathrm{e}}$ siècle est majoritairement le fait d'artistes français, il en existe aussi une non négligeable en Angleterre, ainsi qu'en Allemagne. Elle se situe en Bavière autour de l'École de Munich pendant une courte période : de 1870 à 1882, c'est-à-dire la première décennie de l'Empire allemand. À partir de 1840, autour des personnalités d'Adolf Menzel (1815-1905) et surtout de Karl von Piloty (1826-1886) se mit en place, en réaction contre l'idéalisme des Nazaréens et sous les influences belges et françaises, une conception très narrative de la peinture d'histoire. En tant que professeur à l'Académie de Munich, Piloty assura par son enseignement la pérennité de ce mouvement. Parmi ses élèves, le fameux Viennois Hans Makart mais aussi le Thuringien Ernst Meisel. En 1873, ce dernier représenta d'une façon très théâtrale et pleine de pathos la séparation de Louis XVI de sa famille à la prison du Temple le 26 septembre 1792. Le tableau fut aussitôt exposé par des Sociétés des amis des arts à Munich en 1873 et en Autriche l'année suivante, avant d'être acquis en 1875 par une 
association allemande soutenant la peinture d'histoire, non sans un débat politique et nationaliste. Remarquée pour ses qualités plastiques et d'expression de la douleur des personnages, cette œuvre de Meisel qui embarrassait sans doute ses propriétaires parce qu'elle n'était pas "politiquement correcte », fut finalement revendue rapidement en 1881 à l'un des correspondants de la Verbindung für historische Kunst, la Société des amis des arts de Bâle, ville germanique mais étrangère où le tableau ne pouvait plus faire l'objet d'une controverse aussi vive. En revanche, dans le contexte de Vizille, cette œuvre superbe retrouve sa pleine dimension de chambre d'écho de la Révolution française dans une période historique charnière de l'histoire allemande.

Remy-Furcy Descarsin (actif à Paris dans le dernier tiers du XVIII siècle).

Portrait d'un garde national et de sa femme.

1791.

S.D. en bas à gauche : ft Descarsin/ peintre de Monsieur/ frère du Roy/1791.

Huile sur toile.

H. 0,905 m. ; L. 0,73 m.

Inv. MRF 2004-14.

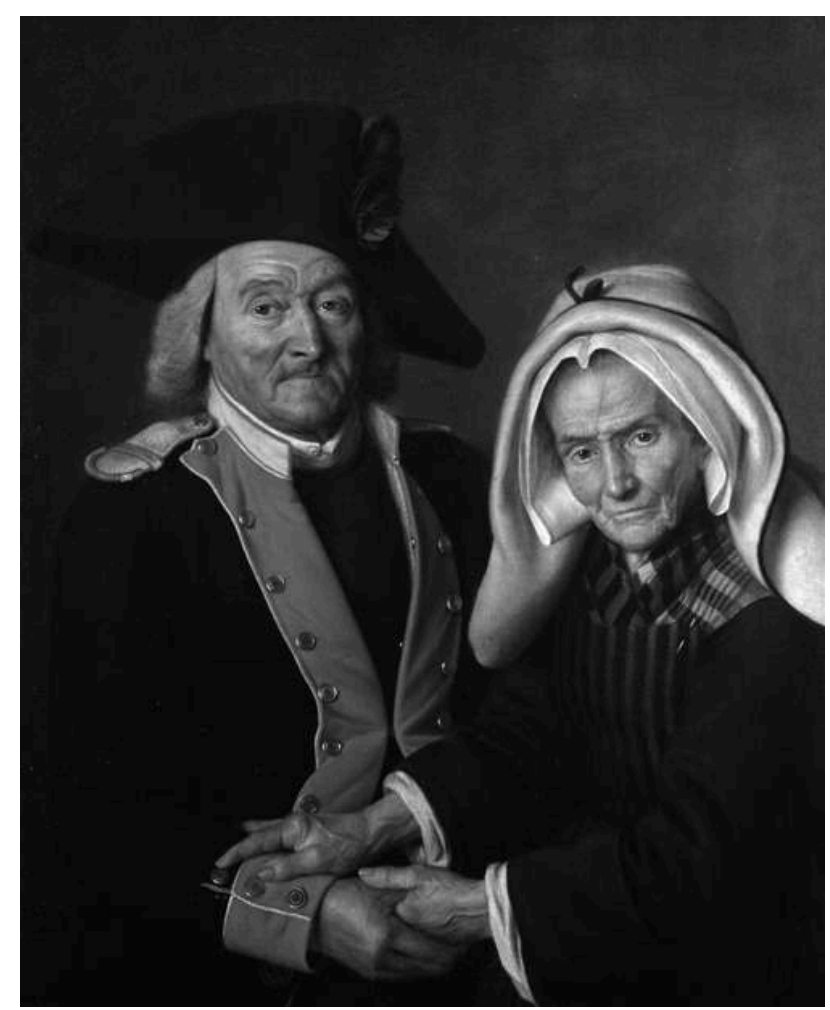

4 Remy-Furcy Descarsin, portraitiste actif à Paris dans le dernier tiers du XVIII ${ }^{\mathrm{e}}$ siècle, a tenté vainement d'entrer à l'Académie en 1789, à laquelle il proposa son autoportrait qui fut refusé. Bien peu d'éléments permettent d'avoir une idée exacte de sa carrière, sinon qu'il évoluait dans un milieu en contact avec l'aristocratie, où la musique tenait une grande place, puisque ses deux sœurs jouaient d'un instrument. D'après l'inscription du tableau, Descarsin se qualifie lui même de "peintre de Monsieur frère du roi ", c'est-à-dire le comte de Provence. Une telle revendication dans le contexte de l'année 1791 est antérieure à la fuite réussie de ce prince, concomitante de celle malheureuse du roi en juin. Aussi le double portrait, récemment réapparu en vente publique, est une véritable découverte d'autant plus que la vérité du rendu psychologique du couple âgé et l'extraordinaire réalisme de la facture, plaident pour un 
portraitiste de qualité. Descarsin qui devait être familier avec les modèles, traduit avec sensibilité à la fois une réalité intime (couple uni, jovialité du mari, dignité et profond dévouement de l'épouse), sociale (la ménagère et le concierge, petit boutiquier ou bien encore artisan) et enfin historique avec le costume de garde national. Ce dernier, porté cependant de façon négligée soulignant ainsi la destination privée du tableau, traduit surtout la fierté de cet homme d'être à sa manière un acteur des temps nouveaux.

Émile Mascré (actif à Paris vers les années 1830-1840).

Capet, Lève toi !

Vers 1833-1835

S. en bas à gauche sur le dallage : ÉMILE MASCRÉ

Huile sur toile.

H. 2,19 m. ; L. 2,56 m.

Inv. MRF 2005-1.

Ancienne collection Domecq (Andalousie)

Achat avec le concours du FRAM Rhône-Alpes.

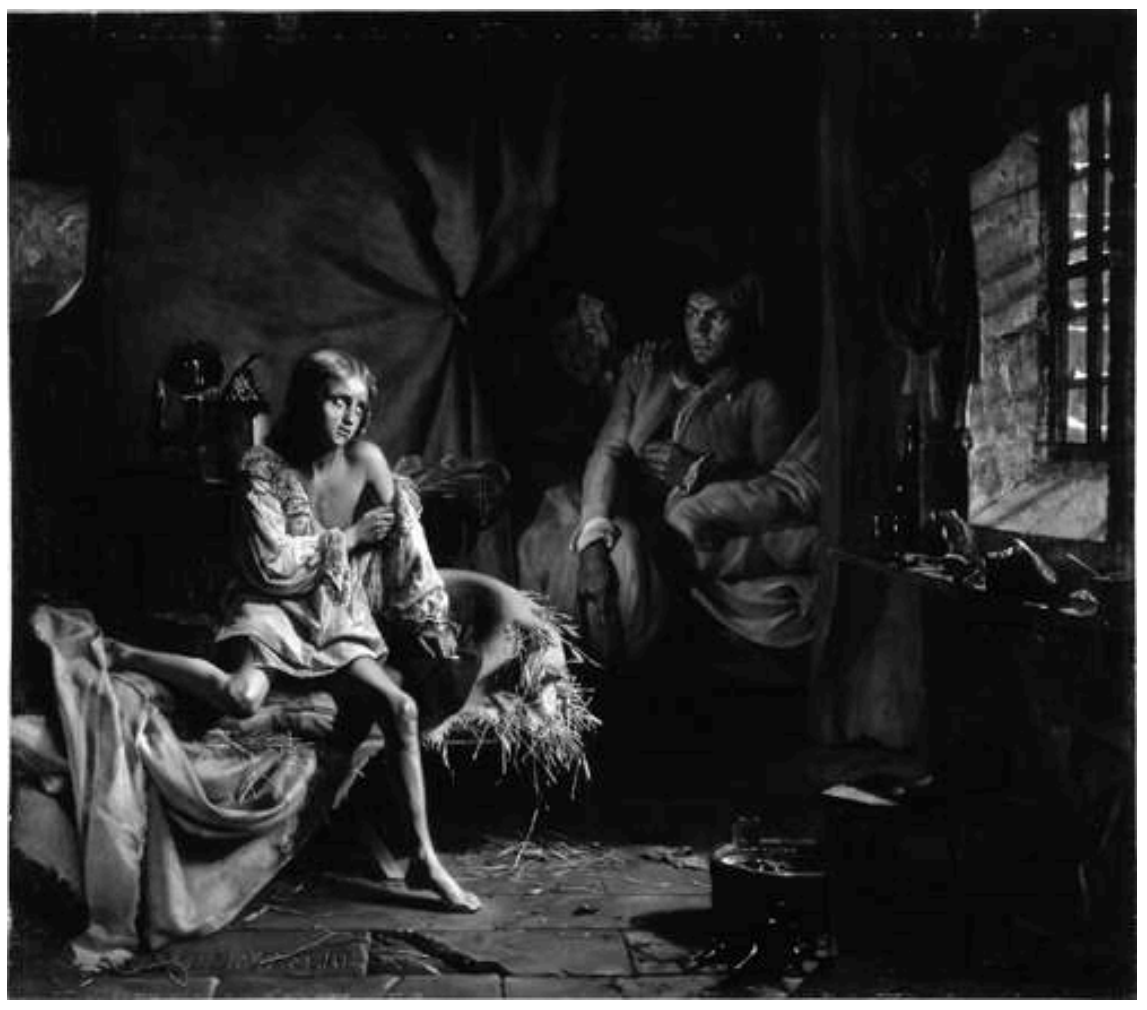

5 Émile Mascré est un peintre très peu documenté dont on sait seulement qu'il participa au Salon dans les années 1830 avec surtout des portraits, et qu'il reçut en 1837 la modeste commande de la copie d'un autoportrait de Van Dyck pour Versailles. Ces maigres indications ne laissent pas supposer l'existence d'un tableau aussi fort et maîtrisé que Capet, Lève toi ! qui aurait pu être un tableau de Salon. Il a été gravé par Jean-François Boisselat. La scène correspond à ce que Mascré pouvait connaître de la mort du dauphin à travers le journal de Cléry, valet de chambre de Louis XVI, publié à Paris en 1816. Selon ce mémoire royaliste, le cordonnier Simon, "un bourreau scélérat », et sa femme qui avaient la garde de l'enfant, le réveillaient plusieurs fois par nuit pour bien s'assurer de sa présence. Cette vision terrible des rapports entre Simon et le dauphin ne correspond pas à la réalité historique qui est celle d'un Simon attentif et protecteur. Tout le génie de Mascré, outre son excellent métier, est de donner à cette 
scène sordide une force extraordinaire grâce à une composition audacieuse, un effet de lumière très puissant, un réalisme descriptif poussé (notamment dans les instruments de cordonnerie) et aux expressions des trois personnages. Tout cela donne un tableau sans concession et troublant par la sourde violence qu'il dégage. De surcroit, le tableau évoque magistralement un épisode de la Révolution à partir duquel s'est développé le mythe de l'héritier royal martyr, à l'instar des enfants d'Edouard pour l'Angleterre (Paul Delaroche, 1830, Musée du Louvre), qui a nourri jusqu'à nos jours de nombreuses polémiques autour du destin de la famille royale et de la violence révolutionnaire.

Joseph-Emmanuel van den Büssche (Anvers, 1837 - Boitsfort, près de Bruxelles, 1908). Le Peintre David dessinant Marie-Antoinette conduite au supplice, 1793.

1900.

S.D. en bas à droite : Emmanuel van den Büssche 1900.

Huile sur toile.

H. 1,005 m. ; L. 0,805 m.

Inv. MRF 2004-18.

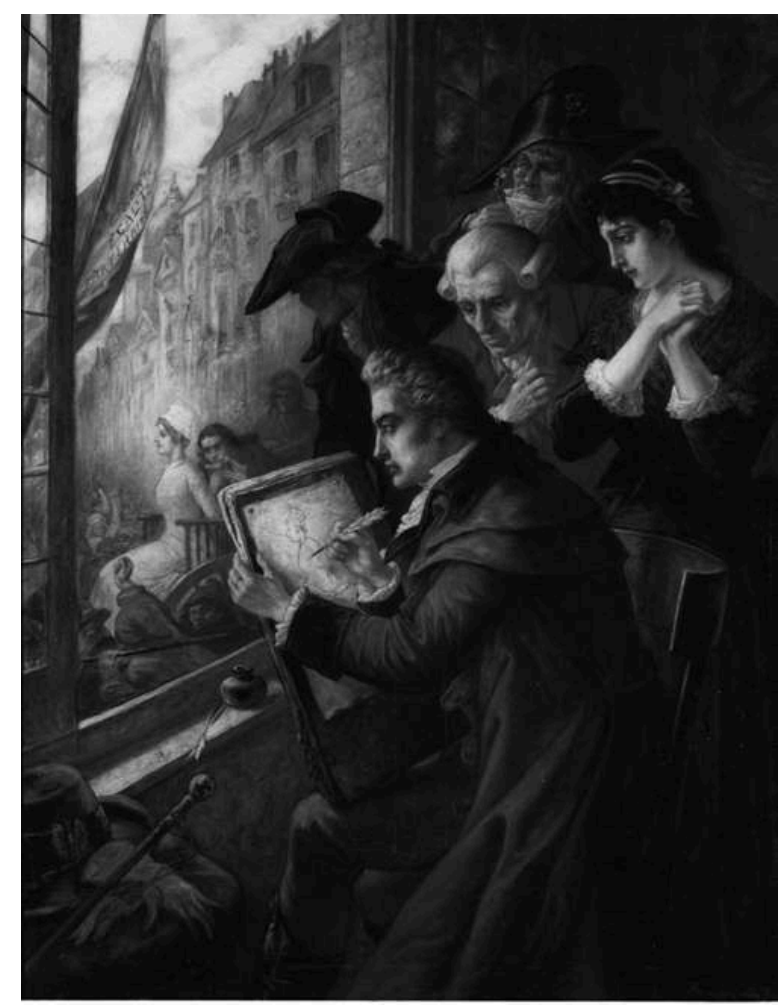

6 La découverte du tableau de van den Büssche vient à sa manière enrichir le dossier déjà fourni du célèbre dessin, aujourd'hui conservé au Louvre, que David aurait exécuté en toute hâte lors du passage de Marie-Antoinette allant au supplice, le 16 octobre 1793 , dont l'attribution est toujours débattue. D'après une annotation manuscrite portée sur l'original, on sait que David se trouvait au balcon d'un immeuble situé sur le passage du convoi, en compagnie de la femme du conventionnel régicide Marc-Antoine Jullien, député de la Drôme. C'est de Madame Jullien que le célèbre collectionneur contemporain de la Révolution, Jean-Louis Soulavie, tenait le dessin. Au XIX ${ }^{\mathrm{e}}$ siècle, cette scène n'a inspiré curieusement que François-Auguste Trichon qui montre David à une fenêtre dessinant le convoi qui n'est pas représenté. Van den Büssche a choisi de se placer à l'intérieur de la pièce afin de mieux faire voir tous les protagonistes sur des plans superposés. Comme toujours, les artistes étrangers représentent des scènes liées 
aux malheurs de la famille royale, mais l'originalité du choix de cet artiste belge est de mettre en avant un authentique révolutionnaire, David, dont l'exil à Bruxelles de 1816 à 1825, a marqué la vie artistique de cette capitale. L'essentiel de l'activité de van den Büssche s'est de fait déroulée à Bruxelles où il enseigna et reçut de nombreuses commandes.

Augustin Pajou (Paris, 1730 - Paris, 1809)

Buste de Maurice Riban.

1792.

S.D. sur la tranche : PAR PAJOU CITOYEN DE PARIS/ A MONTPELLIER L'AN 1792.

Terre cuite.

H. 0,50 m. ; L. 0,42 m. ; P. 0,31 m.

Inv. MRF 2006-1.

Achat avec le concours du FRAM Rhône-Alpes.

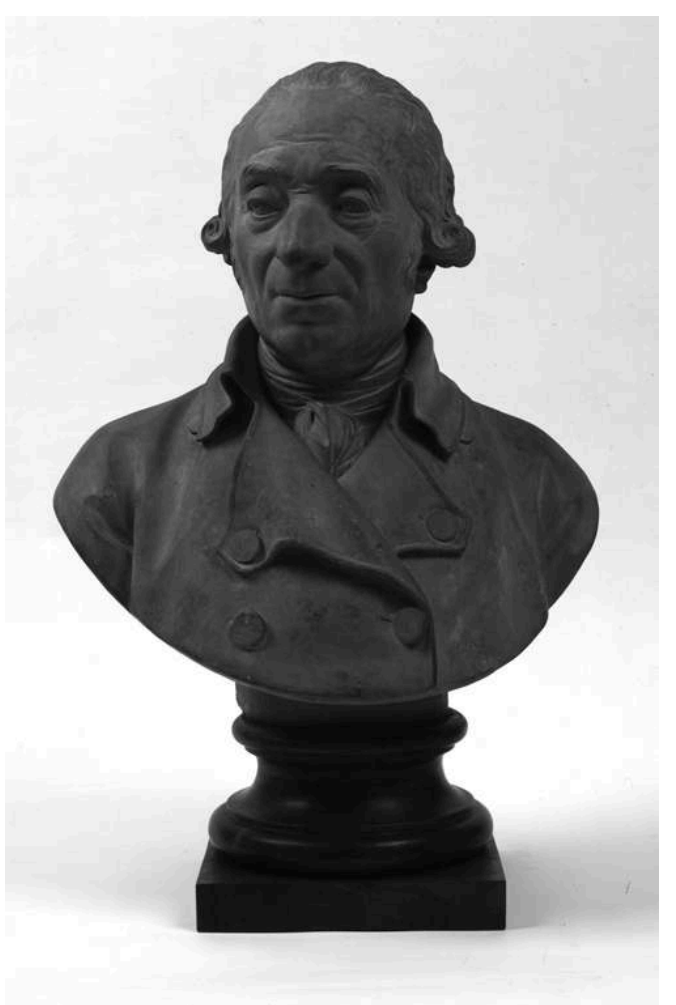

7 Le séjour d'Augustin Pajou, à la fin de sa carrière, à Montpellier entre octobre 1792 et octobre 1794 est un sujet bien connu depuis les travaux d'Henri Stein en 1904, approfondis et réactualisés dans le courant des années 1990. Pendant cette période difficile de la vie de l'artiste (maladie et mort de sa femme) et en regard de l'actualité du pays (instauration de la République, déclaration de guerre, régime de la Terreur), la production $\mathrm{du}$ célèbre sculpteur parisien ne s'est en rien ralentie, comme en témoignent de nombreux bustes dont la quasi totalité est conservée dans des collections publiques françaises et américaines (notamment au Musée Fabre à Montpellier). Ceux de Maurice Riban, chez qui Pajou a vécu pendant ces deux années, et de son fils Jean-Baptiste acquis récemment par Montpellier étaient restés chez leurs descendants. Maurice Riban (1721-1800) était un riche négociant montpelliérain, mécène de la Société des Beaux-Arts de la ville, incontestablement un humaniste qui permit à Pajou de vivre confortablement cette période, en l'introduisant dans les milieux artistiques, mondains et politiques de cette capitale provinciale où il trouva des 
commandes et put faire partager son expérience. Premier des portraits réalisés à Montpellier, celui de Maurice Riban est un très bel exemple de l'art de Pajou, un travail sobre, sincère, alerte et tendu, parfaitement conforme à l'esprit de l'époque. Augustin Pajou et Maurice Riban qui avaient respectivement en 1792, 62 et 71 ans font partie de ces citoyens, hommes des Lumières, vivant pleinement les bouleversements du temps et illustrent de manière éloquente ce que pouvait être la vie artistique en province, au cœur même des événements révolutionnaires.

\section{Bannière de grenadier}

La Constitution ou la Mort

\section{3.}

D. sur le canon : L'AN 4 DE LA LIBERTÉ.

Huile sur toile et manches en bois peint.

H. 1,60 m. ; L. 0,90 m.

Inv. MRF 2003-5.

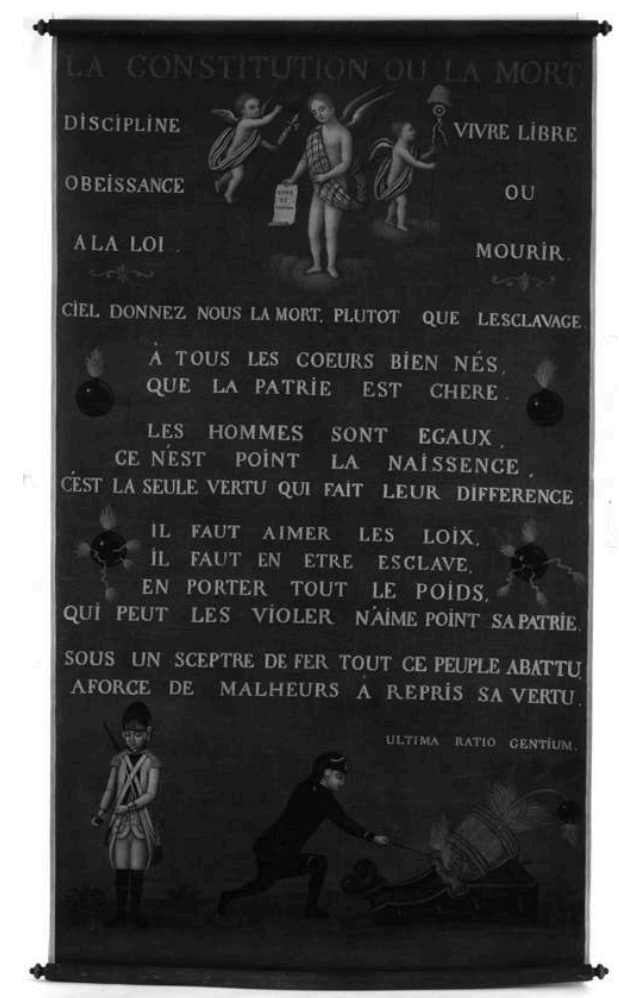

8 Les grenadiers devenus au fil du temps des fusiliers d'élite plutôt que de simples lanceurs de grenade, leur emblème, étaient regroupés en compagnie dans chaque bataillon d'infanterie. En tant que défenseurs de la République sur les champs de bataille, il n'est pas étonnant que les concepteurs de cette bannière exceptionnelle aient mis en avant la constitution votée le 24 juin 1793. En dehors des devises militaires (Discipline, obéissance à la loi) et jacobines (Vivre libre ou mourir), les autres textes inscrits sur la bannière ont été choisis dans l'œuvre de Voltaire. 
Victor-Jean Nicolle (Paris, 1754 - Paris, 1826).

Le Club des patriotes de Toulon.

1793.

S.D. à droite à mi-hauteur sur la façade de l'église : V.J. Nicole/1793.

Plume et encre noire, lavis brun

H. 0, 235 m. ; L. 0, 44 m.

Inv. MRF 2004-12

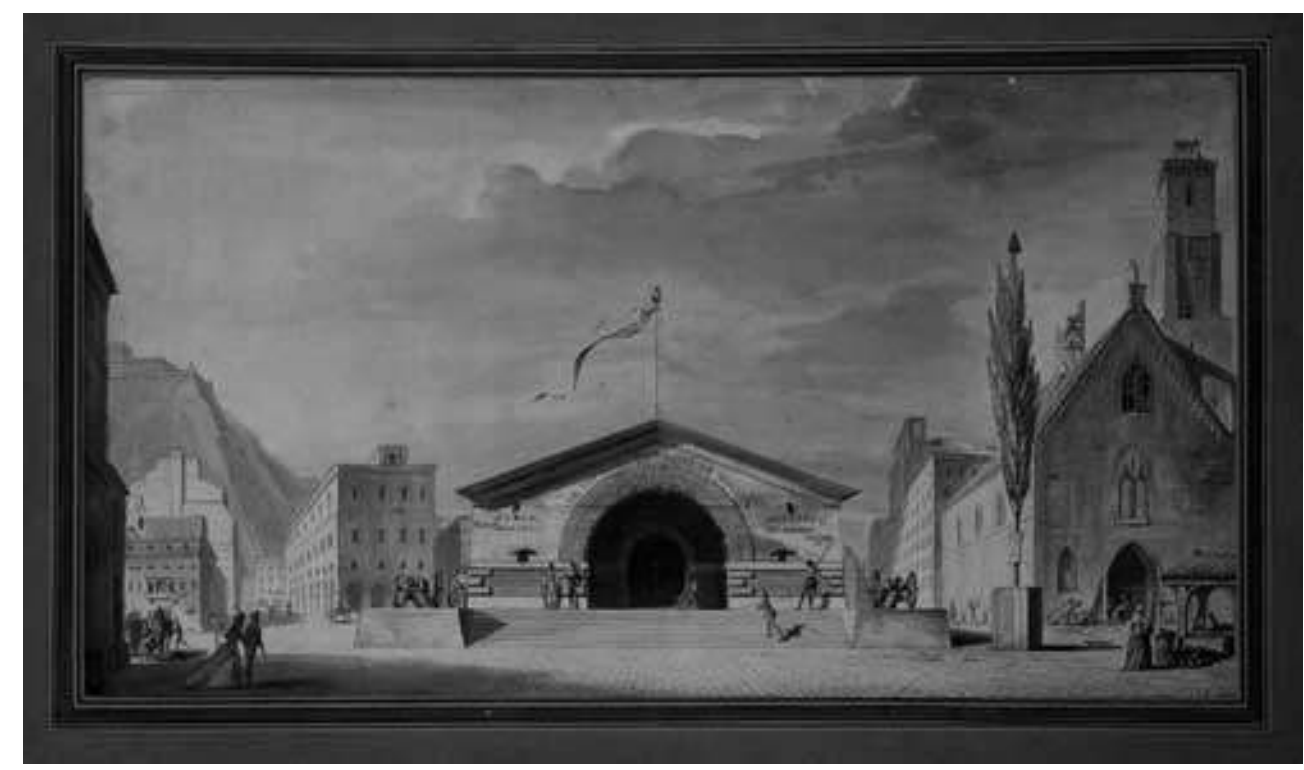

Les représentations des bâtiments abritant les clubs patriotiques pendant la période révolutionnaire sont assez rares et ne concernent qu'exceptionnellement ceux de province. Le dessin très détaillé de Nicolle représente un club de Toulon dont l'architecture moderne s'inspire visiblement de Ledoux, situé sur une place avec un arbre de la Liberté et une église, probablement conventuelle, destinée à la vente suivant l'affiche au dessus de la porte d'entrée. Il serait nécessaire de faire le point sur les clubs toulonnais, leurs locaux et l'urbanisme ancien de cette ville très mutilée par la dernière guerre pour apprécier le degré de réalisme de ce dessin qui pourrait tout aussi bien être un projet. En l'état actuel des connaissances, la biographie de Nicolle, grand dessinateur d'architectures et de vues urbaines et bien évidemment, comme l'implique ce genre, grand voyageur, n'est pas assez détaillée pour savoir avec certitude s'il se trouvait à Toulon en 1793.

Manufacture royale de Sèvres.

Médaillons avec les profils de Louis XVI et Marie-Antoinette.

Avant 1787

Marque sur la tranche de chaque profil : sevres. ; au revers de Louis XVI : $M$; de même pour MarieAntoinette : Li.

Biscuit à l'imitation de Wedgwood.

D. 0,92 m. ; P. 0,15 m.

Inv. MRF 2003-10-1 et 2. 

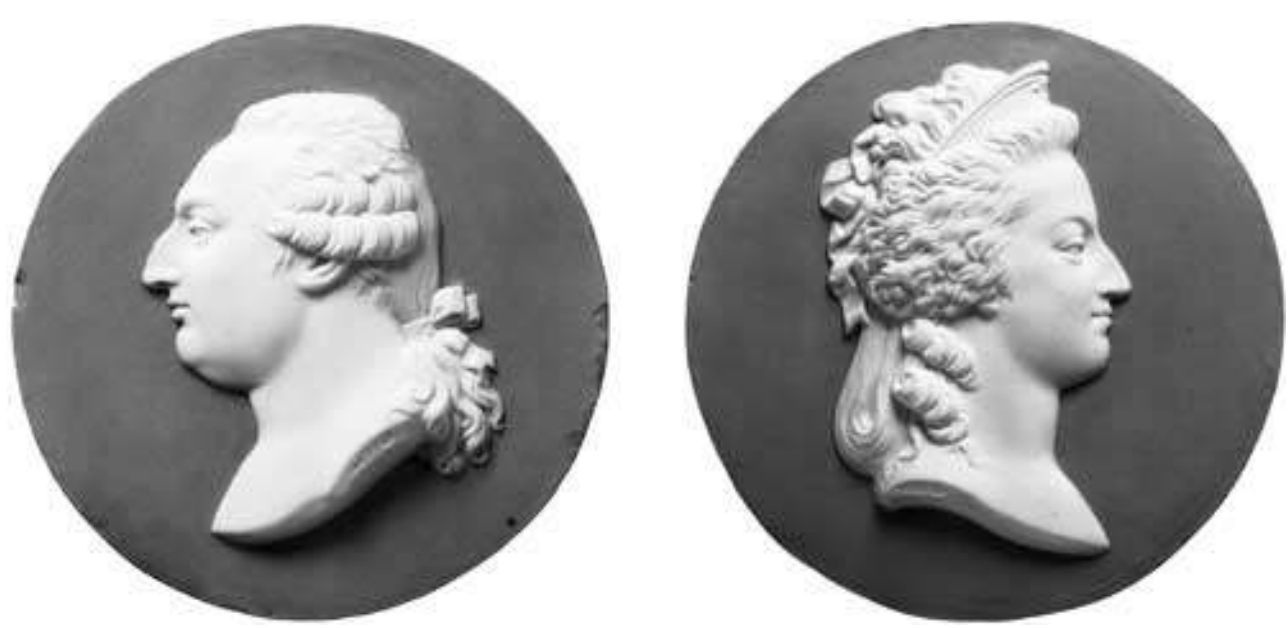

10 Un des moyens de diffusion des portraits des souverains était les médaillons en biscuit qui furent produits en abondance par la manufacture royale de Sèvres de 1774 à 1792, à partir de modèles variés fournis par de nombreux artistes dont bon nombre n'ont pas encore été identifiés. Les deux médaillons de Vizille sont datables de la première moitié des années 1780 à cause du procédé utilisé : le fond bleu à l'imitation de la manufacture de Wedgwood a été appliqué sur la surface alors que la porcelaine était encore crue. Ce n'est qu'à partir de 1787 que Sèvres réussira à teinter la masse.

\section{Bonbonnière de poche ou Tabatière avec un décor commémorant la conquête de la Hollande}

1795.

S. D. en bas de la miniature vers la droite : JJDe Gault fece l'an 3.

Écaille brune, or, miniature sous verre en camaïeu de bleu sur ivoire formant le décor du couvercle. H. 0,21 m. ; D. 0,81 m.

Inv. MRF 2004-15.

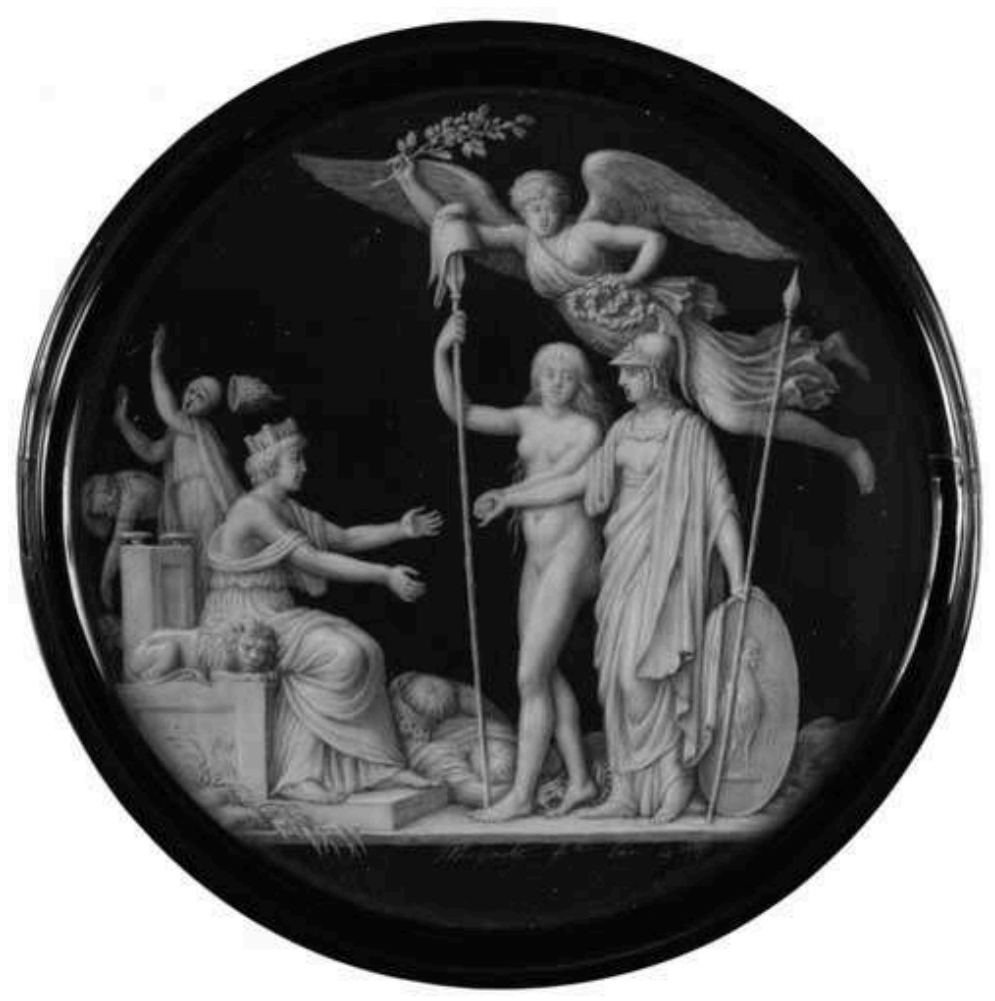


11 La collection de tabletterie du musée s'est développée depuis son origine par des acquisitions régulières et surtout grâce à deux importants dépôts en 1999 et en 2004 du Musée des Arts décoratifs à Paris (19 tabatières au total). Aucune de ces pièces cependant ne comporte un décor en miniature comparable à celui-ci daté et signé Jacques-Joseph De Gault (?, vers 1738- ?, après 1812). Ce dernier est l'auteur de celui de même facture ornant le célèbre "coffre aux diamants" ou serre-bijoux de 1787, commandé par Marie-Antoinette, aujourd'hui dans la chambre de la reine à Versailles. Carlo Jeannerat qui est le premier a avoir bien identifié en 1935 les différentes personnalités des artistes portant le nom de " Gault », a relevé que Jacques-Joseph était actif entre 1771 et l'an III. Pour cette dernière date, peut-être faisait-il allusion à une miniature comparable à celle aujourd'hui à Vizille dont le sujet allégorique se réfère à la récente conquête de la Hollande pendant l'hiver 1794-1795, mettant fin au régime du stathouder Guillaume d'Orange et établissant la République Batave.

\section{AUTEUR}

\section{ALAIN CHEVALIER}

Conservateur en chef du musée de la Révolution française, BP 38220 Vizille, musee.revolution@cg38.fr 